\title{
Aislados clínicos de Pseudomonas aeruginosa productores de $\beta$-lactamasa de espectro extendido en un centro privado de Córdoba
}

\author{
Andrea L. Piersigilli, M. Cecilia Enrico, M. Eugenia Bongiovanni, \\ Liliana E. Bilbao, Gustavo Martínez y Elizabeth M. Ledesma
}

\section{Clinical isolates of Pseudomonas aeruginosa producers of extended spectrum $\beta$-lactamases at a private institution in Cordoba}

Extended spectrum $\beta$-lactamases (ESBL) are capable of inhibiting the action of extended spectrum cephalosporins and monobactams. The objective of this work is to describe six isolates of ESBL producing Pseudomonas aeruginosa, retrieved from intensive care patients. The susceptibility test was performed by diffusion. For the phenotypic detection of ESBL, the following was assessed: the difference between ceftazidime ceftazidime/clavulanic acid (CAZ-CAC) and the synergy between imipenem-ceftazidime (IMI-CAZ) and cefepime-ceftazidime/clavulanic acid -ceftazidime (FEP-CAC-CAZ). The presence of metallo- $\beta$-lactamases (MBL) was discarded through the double disc imipenem-EDTA/mercaptoacetic-meropenem (IMI-EDTA/SMA-MER) method. Molecular characterization of ESBL was performed by polimerase chain reaction (PCR) with blaGES primers. Synergy IMI-CAZ was observed in the studied strains; ESBL type GES was confirmed in five of them. The strategic location of the discs and the evaluation of alert signals for the detection of ESBL is essential, thus contributing to the correct recommendation of treatment in the clinical report.

Key words: Pseudomonas aeruginosa, extended spectrum $\beta$-lactamase, GES.

Palabras clave: Pseudomonas aeruginosa, $\beta$-lactamasas de espectro extendido, GES.

\section{Introducción}

D entro de los bacilos gramnegativo no fermentadores de glucosa, Pseudomonas aeruginosa es el patógeno mejor conocido ${ }^{1}$. Puede provocar infecciones graves como: neumonía, infecciones del tracto urinario y bacteriemia. Estas infecciones suelen ser difíciles de tratar debido a la resistencia intrínseca que presenta $P$. aeruginosa y a su extraordinaria capacidad de adquirir mecanismos de resistencia. Ceftazidima (CAZ) e imipenem (IMI) representan la mejor opción contra infecciones por Pseudomonas sp, ya sea como monoterapia o terapia combinada ${ }^{2,3}$. Sin embargo, la resistencia adquirida contra estos agentes constituye el mayor desafío para el tratamiento de este microorganismo, especialmente cuando está asociada con resistencia a otra clase de antimicrobianos, tales como aminoglucósidos y fluoroquinolonas ${ }^{3,6}$.

Varios mecanismos pueden contribuir para adquirir resistencia a $\beta$-lactámicos en $P$. aeruginosa, incluyendo la producción de $\beta$-lactamasas, la sobre-regulación de sistemas de eflujo, y una disminución de la permeabilidad de la membrana externa ${ }^{4}$. Con respecto a la producción de $\beta$-lactamasas, uno de los principales mecanismos de resistencia a cefalosporinas de amplio espectro es la des-represión mediada por la enzima cromosomal AmpC, que es intrínseca de esta bacteria. No obstante, la adquisición de elementos genéticos móviles que codifican para $\beta$-lactamasas de espectro extendido (BLEE), tales como serino $\beta$-lactamasas de clase A y D de Ambler y las metalo- $\beta$-lactamasas (MBL) de clase B de Ambler, son importantes mecanismos involucrados en la resistencia en $P$. aeruginos $a^{3-4,6-7}$.

Las BLEE son enzimas capaces de inactivar penicilinas, cefalosporinas de espectro extendido y monobactámicos ${ }^{8}$. Han sido extensamente reportadas en miembros de la familia Enterobacteriaceae desde los años 80 y hace poco tiempo se describieron cinco tipos de BLEE clase A de Ambler (TEM, SHV, PER, VEB, GES/IBC) en $P$. aeruginos $a^{6,8-11}$. Hasta ahora se han encontrado en un número de áreas geográficas limitadas ${ }^{3,9,12-14}$ y sólo recientemente han sido reportadas en Argentina ${ }^{15}$.

La BLEE tipo GES-1 se identificó por primera vez en una cepa de Klebsiella pneumoniae en la Guayana Francesa en el año 1998 y, subsecuentemente, en Francia9 ${ }^{9,13,16}$. Los genes bla GES se encuentran mayormente como "cassettes" genéticos insertados dentro de integrones clase $1^{6,8}$. Actualmente se conocen nueve tipos de $\beta$-lactamasas
Sanatorio Privado Aconcagua, Córdoba, Argentina Servicio de Bacteriología (ALP, MCE, MEB, LEB y EML)

Sanatorio Privado Jardín y Santo Tomás, Córdoba, Argentina Servicio de Infectología (GM)

Recibido: 22 de marzo de 2009 Aceptado: 4 de junio de 2009

Correspondencia a: Andrea L. Piersigilli apiersi@hotmail.com 
GES $^{11,17}$. Desafortunadamente, en varios artículos hubo confusiones en la nomenclatura con respecto a la numeración de los genes ${ }^{18}$. Hasta abril de 2006, cuatro tipos de GES (GES-1,-2,-8 y -9) habían sido detectadas en $P$. aeruginosa y, recientemente, en China se reportó el descubrimiento de GES-5 en un aislado de $P$. aeruginosa CAZ resistente ${ }^{11}$.

Las enzimas de la familia GES hidrolizan principalmente CAZ y son susceptibles de inhibir por IMI. Su presencia disminuye notablemente las opciones terapéuticas, por ello es indispensable su detección ${ }^{2,8}$.

Nuestro objetivo es describir seis aislados de P. aeruginosa productores de BLEE, recuperados en un sanatorio privado de Córdoba, Argentina, entre diciembre de 2006 y diciembre de 2007.

\section{Material y Métodos}

Se realizó un estudio descriptivo y prospectivo en un sanatorio privado de la provincia de Córdoba, Argentina, en el período comprendido entre diciembre de 2006 y diciembre de 2007.

Los datos analizados fueron extraídos a partir de los registros del laboratorio de Bacteriología y de las historias clínicas de cada paciente.

Todos los aislados sometidos a análisis fueron jerarquizados por considerarse $P$. aeruginosa el agente causal de la infección.

Las cepas provenían de cinco pacientes (A, B, C, D y E) internados en una unidad de terapia intensiva (UTI). Las mismas fueron recuperadas de orina (pacientes A, B, C), secreción de herida quirúrgica (paciente B), y a partir de muestras respiratorias: mini-lavado broncoalveolar (minibal) (paciente D) y esputo (paciente E). La identificación de las cepas se realizó según pruebas bioquímicas convencionales.

Pruebas de susceptibilidad. Se realizaron por difusión con discos (método de Kirby-Bauer). Se testearon: ticarcilina (TIC) $(75 \mu \mathrm{g})$, piperacilina (PIP) $(100 \mu \mathrm{g})$,

Figura 1. Detección fenotípica de Pseudomonas aeruginosa productora de BLEE GES-like por el método de sinergia entre IMI-CAZ, correspondiente al aislado $B$ (Distancia de borde a borde de $15 \mathrm{~mm}$ ).

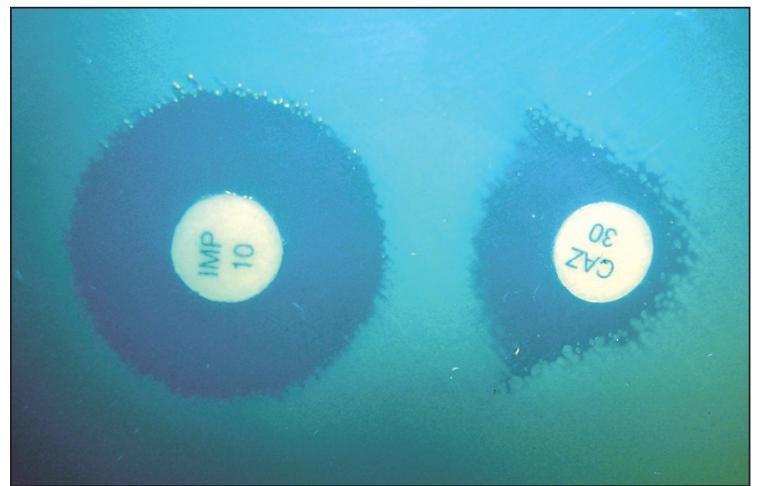

piperacilina/tazobactam (TAZ) $(110 \mu \mathrm{g})$, cefoperazona (CPZ) $(75 \mu \mathrm{g})$, CAZ $(30 \mu \mathrm{g})$, cefepima (FEP) $(30 \mu \mathrm{g})$, aztreonam (AZT) $(30 \mu \mathrm{g})$, IMI $(10 \mu \mathrm{g})$, meropenem (MER) $(10 \mu \mathrm{g})$, gentamicina (GEN) $(10 \mu \mathrm{g})$, amikacina $(\mathrm{AKN})(30 \mu \mathrm{g})$, ciprofloxacina (CIP) $(5 \mu \mathrm{g})$ y colistina (COL) $(10 \mu \mathrm{g})^{3}$. Se utilizó además disco de CAC (ceftazidima/ác. clavulánico) $(30 \mu \mathrm{g} / 10 \mu \mathrm{g})$ para maximizar la información obtenida del antibiograma. Los puntos de corte utilizados para interpretar la sensibilidad de cada antimicrobiano fueron los recomendados por el Clinical Laboratory Standard Institute vigente el año del estudio (CLSI M2-A9, M100-S17, 2007).

Estudios complementarios. Los métodos de tamizaje para la detección de BLEE fueron:

- Comparación del diámetro de los halos de inhibición de CAZ y de la combinación CAC. Una diferencia $\geq$ a $5 \mathrm{~mm}$ entre CAZ y CAC se interpretó como probable presencia de BLEE.

- Sinergia entre IMI y CAZ. Los discos se colocaron a una distancia de $15 \mathrm{~mm}$ de borde a borde. Se consideró sinergia positiva al agrandamiento del halo de inhibición de CAZ en la zona adyacente al disco de IMI.

- Sinergia entre FEP-CAC-CAZ, donde la distancia entre los discos fue de 15 a $20 \mathrm{~mm}$ de borde a borde. Un agrandamiento en los halos de inhibición de FEP y CAZ en la zona adyacente al disco de CAC fue interpretado como posible presencia de $\mathrm{BLEE}^{10,15}$.

La búsqueda de MBL se determinó evaluando la presencia o ausencia de sinergia entre los discos de IMI, EDTA/SMA (750 $\mu \mathrm{g}$ de EDTA más $2.000 \mu \mathrm{g}$ de mercaptoacético de sodio) y MER; la distancia entre los discos fue de $10 \mathrm{~mm}$ de borde a borde ${ }^{19}$.

Caracterización molecular. La caracterización molecular del fenotipo BLEE se realizó por reacción de polimerasa en cadena (RPC) con cebadores específicos blaGES en el Servicio Antimicrobianos, Departamento de Bacteriología, INEI-ANLIS “Dr. Carlos G. Malbrán”.

\section{Resultados}

Durante el período estudiado se recuperaron seis cepas de $P$. aeruginosa provenientes de pacientes hospitalizados. La edad promedio de los pacientes de sexo femenino (A y C) fue de 86 años y de los pacientes de sexo masculino (B, D, E) fue de 66 años.

Los perfiles de susceptibilidad para cada cepa se muestran en la Tabla 1.

En todas las cepas se observó sinergia con IMI-CAZ (Figura 1) y no con FEP-CAC-CAZ. La diferencia CACCAZ fue $\geq 5 \mathrm{~mm}$ en los aislados provenientes de los pacientes A, B, C, y E.

La búsqueda de MBL fue negativa, ya que no se observó sinergia entre IMI-EDTA/SMA-MER. 
Tabla 1. Patrón de susceptibilidad in vitro de Pseudomonas aeruginosa recuperadas en cinco pacientes

\begin{tabular}{|c|c|c|c|c|c|}
\hline Antimicrobianos & \multicolumn{5}{|c|}{ Pacientes } \\
\hline Ticarcilina & R & $\mathrm{R}$ & $R$ & $\mathrm{R}$ & $\mathrm{R}$ \\
\hline Piperacilina & R & R & $\mathrm{R}$ & $\mathrm{R}$ & $\mathrm{R}$ \\
\hline Piperacilina/tazobactam & s & s & S & S & $S$ \\
\hline Cefoperazona & R & R & $\mathrm{R}$ & $\mathrm{R}$ & $\mathrm{R}$ \\
\hline Ceftazidima & R & $\mathrm{R}$ & $\mathrm{R}$ & $\mathrm{R}$ & $\mathrm{R}$ \\
\hline Cefepima & $S^{*}$ & $1^{*}$ & $S^{*}$ & $1^{*}$ & $S^{*}$ \\
\hline Aztreonam & $S^{*}$ & $1^{*}$ & $1^{*}$ & $1^{*}$ & $S^{*}$ \\
\hline Imipenem & S & S & S & S & $S$ \\
\hline Meropenem & S & S & S & S & $\mathrm{R}$ \\
\hline Gentamicina & $\mathrm{R}$ & $\mathrm{R}$ & $\mathrm{R}$ & $\mathrm{R}$ & $\mathrm{R}$ \\
\hline Amikacina & R & $\mathrm{R}$ & $\mathrm{R}$ & $\mathrm{R}$ & $\mathrm{R}$ \\
\hline Ciprofloxacina & R & $\mathrm{R}$ & $\mathrm{R}$ & $\mathrm{R}$ & $\mathrm{R}$ \\
\hline Colistín & S & $S$ & S & S & $S$ \\
\hline \multicolumn{6}{|c|}{$\begin{array}{l}\text { *Se informa resistente por presencia de BLEE. } \\
\text { S, sensible; R, resistente; I, sensibilidad intermedia. } \\
\text { A, C: cepas recuperadas de orina de los paciente A y C. } \\
\text { B: cepas recuperadas de orina y herida quirúrgica del pac B. } \\
\text { D: cepa recuperada de minilavado bronco-alveolar del paciente D. } \\
\text { E: cepa recuperada de esputo del paciente E. }\end{array}$} \\
\hline
\end{tabular}

En las cepas estudiadas, la BLEE fue confirmada a nivel molecular como tipo GES en los aislados de los pacientes A, B, C, y D.

Se detectó la presencia de BLEE en la cepa proveniente del paciente E; sin embargo, la identificación molecular por RPC resultó negativa con cebadores específicos blaGES. Dicho aislado continúa en estudio.

\section{Discusión}

En las dos últimas décadas la resistencia de $P$. aeruginosa a antimicrobianos ha aumentado notablemente y es en la actualidad un problema creciente. Resulta importante que en cada institución hospitalaria se mantenga una estrecha vigilancia de los perfiles de resistencia de esta bacteria, con el fin de reconocer sus mecanismos de resistencia, su evolución y la forma de transferencia ${ }^{20}$.

El ensayo de sinergia de doble disco con ác. clavulánico y cefalosporinas de espectro extendido ofreció dificultades al ser usado para la detección de BLEE en $P$. aeruginos $a^{9,21}$. Estas dificultades pueden ser el producto de varios factores, entre ellos la resistencia relativa a la inhibición por ác. clavulánico por enzimas tipo GES ${ }^{9,14}$. En los aislados de $P$. aeruginosa analizados no se observó sinergia entre CAZ-CAC-FEP, lo que permitió descartar la existencia de otros tipos de $\mathrm{BLEE}^{10}$. En todos se detectó la presencia de BLEE inhibida por IMI, pero sólo en cinco de ellos fue identificada RPC como GES. Podríamos entonces afirmar que la utilización del método de tamizaje (sinergia IMI-CAZ) resultó adecuado para identificar fenotípicamente la producción de BLEE tipo GES, al menos en las cepas estudiadas.

La no detección de BLEE podría haber conllevado a un error en la lectura e interpretación del antibiograma, ya que las cepas presentaron susceptibilidad o sensibilidad intermedia in vitro a las cefalosporinas de cuarta generación y a aztreonam.

Dada la multiresistencia observada, carbapenémicos y polimixinas se transformarían en las opciones terapéuticas posibles. Las cepas de los pacientes A, B, C y D mostraron halos de inhibición considerables frente a carbapenémicos (datos no mostrados) y ausencia de MBL, por lo que su uso no estaría contraindicado. En el paciente E, cuya BLEE no fue tipo GES, quedó excluida la posibilidad de tratamiento con MER ya que presentó resistencia. Posiblemente esta cepa presente, además de la BLEE, otro mecanismo de resistencia asociado como podría ser hiperproducción de bombas de eflujo ${ }^{4,22}$.

Algunos estudios reportaron que en los aislados con 
BLEE que permanecen sensibles a carbapenémicos, se podría proponer el uso de éstos en combinación con un antimicrobiano no $\beta$-lactámico ${ }^{23}$. En las cepas estudiadas fue difícil recomendar sinergia debido a que en todas se observó resistencia a los agentes no $\beta$-lactámicos testeados.

El régimen antimicrobiano más apropiado para el tratamiento de infecciones debido a cepas de $P$. aeruginosa BLEE positivas aún no se ha establecido, debido a que pocos estudios clínicos se han dirigido hacia este campo de investigación ${ }^{9}$. Se documentó falla de tratamiento con FEP y TAZ en modelos animales (neumonía y BLEE tipo PER) asociado a efecto inóculo in vivo ${ }^{24}$.

Además del correcto informe microbiológico, la detección de estas enzimas en $P$. aeruginosa fue importante para la toma de medidas de control epidemiológico debido al perfil de resistencia que confieren y a la potencial capacidad de dispersión del elemento genético asociado ${ }^{9,14}$.

No se pudo establecer relación epidemiológica entre los aislados, dado que no se realizaron las pruebas moleculares correspondientes para confirmar o descartar un posible brote hospitalario.

Concluimos que resulta indispensable la ubicación estratégica de los discos y la evaluación de las señales de alarma de las pruebas de difusión para la detección de BLEE en $P$. aeruginosa. Si bien los métodos moleculares no están disponibles para los laboratorios de microbiología clínica y la co-existencia de múltiples mecanismos de resistencia condicionan la clásica sinergia con ác. clavulánico, la ubicación estratégica de los discos (IMICAZ) resultó fundamental para poder detectar este tipo de enzimas. Logramos contribuir así a la correcta recomendación del tratamiento antimicrobiano en el informe clínico y a su vez pudimos alertar con respecto a la toma de medidas de control epidemiológico.

Agradecimientos. Al personal del Servicio Antimicrobianos, Departamento de Bacteriología, INEI-ANLIS "Dr. Carlos G. Malbrán", y en especial a Fernando Pasteran por su colaboración incondicional y desinteresada.

\section{Resumen}

Las $\beta$-lactamasas de espectro extendido (BLEE) son capaces de inactivar cefalosporinas de espectro extendido y monobactámicos. El objetivo de este trabajo es describir seis aislados de Pseudomonas aeruginosa productores de BLEE recuperados de pacientes de cuidados intensivos. Las pruebas de sensibilidad se realizaron por difusión. Para la detección fenotípica de BLEE se evaluó: diferencia de diámetro entre los halos de ceftazidima-ceftazidima/ clavulánico (CAZ-CAC) y sinergia entre imipenem-ceftazidima (IMI-CAZ) y cefepima-ceftazidima/clavulánicoceftazidima (FEP-CAC-CAZ). Se descartó la presencia de metalo- $\beta$-lactamasas (MBL) por el método de doble disco imipenem-EDTA/mercaptoacético-meropenem (IMI-EDTA/SMA-MER). La caracterización molecular de BLEE se realizó por reacción de polimerasa en cadena con cebadores blaGES. En las cepas estudiadas se observó sinergia IMI-CAZ; en cinco de ellas se confirmó BLEE tipo GES. Es imprescindible la ubicación estratégica de los discos y la evaluación de señales de alarma para la detección de BLEE, contribuyendo así a la correcta recomendación del tratamiento en el informe clínico.

\section{Referencias}

1.- Vidal F, Mensa J, Almela M, Olona M, Martínez J A, Marco F, et al. Bacteraemia in adults due to glucose non-fermentative Gram negative bacilli other than $P$. aeruginosa. Q J Med 2003; 96: 227-34.

2.- Machain M, Suárez J, Ferreiro D, Ceregido E. Detección de $\beta$-lactamasas de espectro extendido (BLEE) en Pseudomonas aeruginosa (PAE) en un hospital de agudos. Resumen. Congreso SADEBAC 2006 Asociación Argentina de Microbiología.

3.- Pagani L, Mantengoli E, Migliavacca R, Nucleo E, Pollini S, Spalla M, et al. Multifocal detection of multidrug-resistant Pseudomonas aeruginosa producing the PER-1 extendedspectrum $\beta$-lactamase in Northern Italy. J Clin Microbiol 2004; 42 (6): 2523-9.

4.- Livermore D M. Multiple mechanisms of antimicrobial resistance in Pseudomonas aeruginosa: Our worst nightmare? Clin Infect Dis 2002; 34: 634-40.

5.- Sekiguchi J I, Asagi T, Miyoshi-Akiyama T, Kasai A, Mizuguchi Y, Araake M, et al. Outbreaks of multidrug-resistant Pseudomonas aeruginosa in community in Japan. J Clin Microbiol 2007; 45 (3): 979-89.

6.- Dubois V, Poirel L, Marie C, Arpin C, Nordmann P, Quentin C. Molecular characterization of a novel class 1 integron containing blaGES-1 and a fused product of $\mathrm{aac}(3)-I b / a a c\left(6^{\prime}\right)-I b^{\prime}$ gene cassettes in Pseudomonas aeruginosa. Antimicrob Agents Chemother 2002; 46 (3): 638-45.

7.- Pasteran F, Faccone D, Petroni A, Rapoport M, Galas M, Vásquez M, et al. Novel variant (blaVIM-11) of the metallo- $\beta$ lactamase blaVIM family in a GES-1 extended-spectrum- $\beta$-lactamase-producing Pseudomonas aeruginosa clinical isolate in Argentina. Antimicrob Agents Chemother 2005;
49 (1): 474-5.

8.- Pellegrino F, Netto-Dos-Santos K, Riley L, Moreira B. M. blaGES carrying Pseudomonas aeruginosa isolates from a public hospital in Rio de Janeiro, Brazil. Brazilian J Infect Dis 2006; 10 (4): 251-3.

9.- Weldhagen G, Poirel L, Nordmann P. Ambler class A extended-spectrum $\beta$-lactamases in Pseudomonas aeruginosa: Novel developments and clinical impact. Antimicrob Agents Chemother 2003; 47 (8): 2385-92.

10.- Subcomisión de Antimicrobianos SADEBAC/ Asociación Argentina de Microbiología. Caracterización fenotípica de la resistencia a los $\beta$-lactámicos en Pseudomonas aeruginosa y Acinetobacter spp. Disponible en la web: http:// www.aam.org.ar/novedades/Consenso $\% 20$ BNNF\%20Anexo.pdf. [accedido: 03 de diciembre 2006].

11.- Wang Ch, Cai P, Chang D, Zuhuang M. A Pseudomonas aeruginosa isolate producing 
the GES- 5 extended-spectrum $\beta$-lactamase. J Antimicrob Chemother 2006; 57 (6): 1261-2.

12.- Poirel L, Naas T, Nordmann P. Pyrosequencing as a rapid tool for identification of GES-type extended-spectrum $\beta$-lactamases. J Clin Microbiol 2006; 44 (8): 3008-11.

13.- Castanheira M, Mendes R, Walsh T, Gales A, Jones R N. Emergence of the extended-spectrum $\beta$-lactamase GES-1 in a Pseudomonas aeruginosa strain from Brazil: Report from the SENTRY Antimicrobial Surveillance Program. Antimicrob Agents Chemother 2004; 48 (6): 2344-5.

14.- Tato M, Valverde A, Coque T M, Canton R. Pseudomonas aeruginosa multirresistente productora de PER-1 en España. Enferm Infecc Microbiol Clin 2006; 24 (7): 472-3.

15.- Pasteran F, Guerreiro L, Goris V, Sujemeckis A, Faccone D, Campos K, et al. $\beta$-Lactamasa de espectro extendido (BLEE) GES-1 en aislamientos clínicos de Pseudomonas aeruginosa de Argentina: Evaluación de métodos fenotípicos para su detección. Resumen. XVII Congreso
Latinoamericano de Microbiología. X Congreso Argentino de Microbiología. 2004 Asociación Argentina de Microbiología.

16.- Poirel L, Le Thomas I, Naas T, Karim A, Nordmann P. Biochemical sequence analysis of GES-1 a novel class A extended- spectrum $\beta$-lactamase, and class 1-integron In52 from Klebsiella pneumoniae. Antimicrob Agents Chemother 2000; 44 (3): 622-32.

17.- Da Fonseca L E, Vieira V, Cipriano R, Cipriano R, Paulo Vicente A C. Emergence of bla-GES-5 in clinical-only-sensitive (COS) Pseudomonas aeruginosa strain in Brazil. J Antimicrob Chemother 2007; 59: 576-7.

18.- Lee S H, Jeong S H, Wachino J, Arakawa Y, Poirel L, Nordmann P. Nomenclature of GEStype extended-spectrum $\beta$-lactamases [letter]. Antimicrob Agents Chemother 2005; 49 (5): 2148-50.

19.- Lee K, Lim Y S, Yong D, Yum J H, Chong Y. Evaluation of the Hodge test and the imipenemEDTA double-disk synergy for differentiating metallo- $\beta$-lactamase-producing isolates of Pseudomonas spp. and Acinetobacter spp. J Clin Microb 2003; 41 (10): 4623-9.
20.- Bodí M, Garnacho J. Pseudomonas aeruginosa: tratamiento combinado frente a monoterapia. Med Intensiva 2007; 31 (2): 83-7.

21.- Cantón R, Loza E, Conejo M del C, Baquero F, Martínez-Martínez L, MENSURA Collaborative Group. Quality control for $\beta$-lactam susceptibility testing with a well-defined collection of Enterobacteriaceae and Pseudomonas aeruginosa strain in Spain. J Clin Microbiol 2003; 41 (5): 1912-8.

22.- Bonomo R, Szabo D. Mechanisms of multidrug resistance in Acinetobacter species and Pseudomonas aeruginosa. Clin Infect Dis 2006; 43: 49-56.

23.- Poirel L, Weldhagen G, Naas T, De Champs C, Dove M G, Nordmann P. GES-2, a class A - $\beta$-lactamase from Pseudomonas aeruginosa with increased hydrolysis of imipenem. Antimicrob Agents Chemother 2001; 45 (9): 2598-603.

24.- Mimoz O, Elheliali N, Léotard S, Jacolot A, Laurent F, Samii K, et al. Treatment of experimental pneumonia in rats caused by a PER-1 extended-spectrum $\beta$-lactamase producing strain of Pseudomonas aeruginosa. J Antimicrob Chemother 1999; 44: 91-7. 